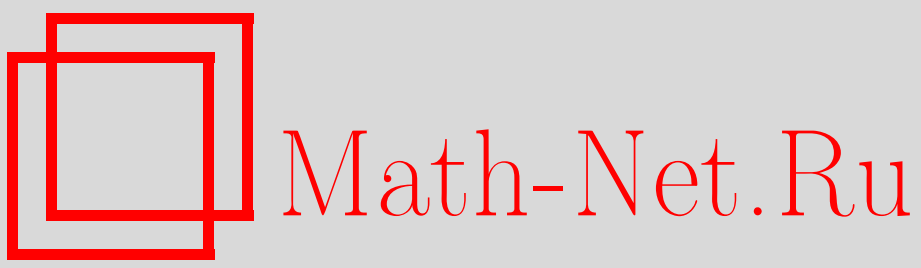

О. Е. Галкин, Бесконечномерные супераналоги формулы Мелера, Матем. заметки, 1996, том 59, выпуск 6, 927-931

DOI: https://doi.org/10.4213/mzm1793

Использование Общероссийского математического портала MathNet.Ru подразумевает, что вы прочитали и согласны с пользовательским соглашением

http://www . mathnet.ru/rus/agreement

Параметры загрузки:

IP: 52.6 .47 .48

26 апреля 2023 г., $17: 37: 53$

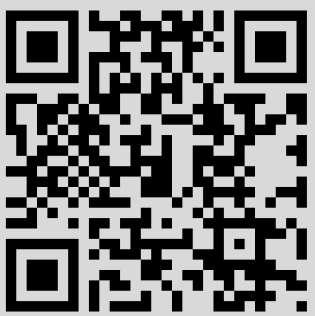




\section{БЕСКОНЕЧНОМЕРНЫЕ СУПЕРАНАЛОГИ ФОРМУЛЫ МЕЛЕРА}

\section{О.Е. Галкин}

В работе найдена явная формула (являющаяся обобщением формулы Мелера, см. [1, с. 34]) решения задачи Коши для бесконечномерного аналога уравнения Шрёдингера гармонического осциллятора относительно функций на суперпространстве. Понятие суперпространства, использованное в работе, было введено в работах [2], [3] (конечномерный случай) и в [4] (бесконечномерный случай). Отметим также работы [5]-[16], посвященные различным аспектам как конечномерного, так и бесконечномерного суперанализа, особенно пионерскую работу $\Phi$.А. Березина (см. [7] и имеющиеся там ссылки).

В настоящей работе используется подход к бесконечномерному суперанализу, описанный в работах [9], [11]. Отметим, что “вещественные" аналоги рассмотренной нами задачи решены в [17], [18]. Супераналоги классических уравнений математической физики изучались также в [10]-[13].

1. Суперпространство. Пусть $\Lambda=\Lambda_{0} \oplus \Lambda_{1}$ - (вещественная) коммутативная банахова супераләебра с тривиальным левым аннулятором (см. [3]) и нормой | | такой, что $|a b| \leqslant|a| \cdot|b|$ (каковы бы ни были $a, b \in \Lambda$ ). Пусть, далее, $G=G_{0} \oplus G_{1}$ и $F$ - (вещественные) градуированные банаховы, $E=E_{0} \oplus V \oplus W$-вещественное сепарабельное гильбертово пространства, причем подпространства $E_{0}$ и $E_{1}=V \oplus W$ ортогональны, а $V$ и $W$ изоморфны. (Здесь и далее прямые суммы пополняются по $\ell_{1}$-норме, для гильбертовых пространств - по $\ell_{2}$-норме.) Фиксируем в $E_{0}, V$ и $W$ ортонормированные базисы. Тогда их объединение $\left\{e_{i}\right\}_{i=1}^{\infty}$ будет базисом в $E$.

Cупермодулем называется банахово пространство $G^{\Lambda}=G \widehat{\otimes} \Lambda$, являюееся пополнением тензорного произведения $G \otimes \Lambda$ по какой-либо кросс-норме $\|\cdot\|$, например, по норме проективной топологии или топологии равностепенно непрерьвной сходимости (см. [19], [20]). В случае сепарабельного гильбертова пространства $E$ в качестве такой нормы будем брать $\ell_{2}$-норму:

$$
\left\|\sum_{i=1}^{n} e_{i} \otimes \lambda_{i}\right\|=\left(\sum_{i=1}^{n}\left|\lambda_{i}\right|^{2}\right)^{1 / 2} .
$$

При этом $E^{\Lambda}$ будет гильбертовым суперпространством (см. [21]). В $G^{\Lambda}$ и $G_{\Lambda}$ естественньм образом вводятся структуры двусторонних модулей над $\Lambda$ и $\Lambda_{0}$ соответственно.

Аналогично определяются пространства

$$
\begin{array}{lll}
\left(E_{\Lambda}\right)_{0}=E_{0} \widehat{\otimes} \Lambda_{0}, & \left(E_{\Lambda}\right)_{V}=V \widehat{\otimes} \Lambda_{1}, & \left(E_{\Lambda}\right)_{W}=W \widehat{\otimes} \Lambda_{1}, \\
\left(E_{\Lambda}\right)_{1}=E_{1} \widehat{\otimes} \Lambda_{1}, & \left(G_{\Lambda}\right)_{0}=G_{0} \widehat{\otimes} \Lambda_{0}, & \left(G_{\Lambda}\right)_{1}=G_{1} \widehat{\otimes} \Lambda_{1} .
\end{array}
$$

Ясно, что $\left(E_{\Lambda}\right)_{1}=\left(E_{\Lambda}\right)_{V} \oplus\left(E_{\Lambda}\right)_{W}$.

Cynерпространствами над $\Lambda$ называются прямые суммы $G_{\Lambda}=\left(G_{\Lambda}\right)_{0} \oplus\left(G_{\Lambda}\right)_{1}$ и $E_{\Lambda}=\left(E_{\Lambda}\right)_{0} \oplus\left(E_{\Lambda}\right)_{1}$. Элементы $E_{\Lambda}$ будем записывать в виде $X=(x, \theta, \bar{\theta})$, где $x=\left(x_{1}, \ldots, x_{n}, \ldots\right) \in\left(E_{\Lambda}\right)_{0}, \theta=\left(\theta_{1}, \ldots, \theta_{n}, \ldots\right) \in\left(E_{\Lambda}\right)_{V}, \bar{\theta}=\left(\bar{\theta}_{1}, \ldots, \bar{\theta}_{n}, \ldots\right)$ $\in\left(E_{\Lambda}\right)_{W}$ 
Суперскалярным произведением $(X, Y)$ произвольных элементов $X=(x, \theta, \bar{\theta})$, $Y=(y, \xi, \bar{\xi})$ из $E_{\Lambda}$ назьвается величина $\sum_{i=1}^{\infty}\left(x_{i} y_{i}+\theta_{i} \bar{\xi}_{i}+\xi_{i} \bar{\theta}_{i}\right)$. (При этом $(X, Y) \in \Lambda_{0},|(X, Y)| \leqslant\|X\| \cdot\|Y\|,(X, Y)=(Y, X)$.)

2. Линейные операторы. Пусть $\operatorname{Hom}\left(F^{\Lambda}, G^{\Lambda}\right)$ - пространство $\Lambda$-линейных справа непрерывных отображений $F^{\Lambda}$ в $G^{\Lambda}$, наделенное равномерной операторной нормой $\|\cdot\| ; \operatorname{Hom}(F, G)$ - подмножество всех таких элементов $A \in \operatorname{Hom}\left(F^{\Lambda}, G^{\Lambda}\right)$, что $A(F) \subset G ; \operatorname{Hom}_{0}(F, G)=\operatorname{Hom}\left(F_{0}, G_{0}\right) \oplus \operatorname{Hom}\left(F_{1}, G_{1}\right)$, $\operatorname{Hom}_{1}(F, G)=\operatorname{Hom}\left(F_{0}, G_{1}\right) \oplus \operatorname{Hom}\left(F_{1}, G_{0}\right), \operatorname{Hom}_{\Lambda}(F, G)$ - соответствующее ему суперпространство (пополнение взято относительно нормы пространства $\left.\operatorname{Hom}\left(F^{\Lambda}, G^{\Lambda}\right)\right)$.

Линейными операторами $B$ на $F_{\Lambda}$ со значениями в $G_{\Lambda}$ называются ограничения на $F_{\Lambda}$ операторов из $\operatorname{Hom}_{\Lambda}(F, G)$, а также сами эти операторы. Каждый линейный оператор $B$ имеет четыре компоненты $B_{i j}:\left(E_{\Lambda}\right)_{i} \rightarrow\left(G_{\Lambda}\right)_{j} ; i, j=0,1$.

Оператором Гильберта-Шмидта назьвается линейный оператор $B$ с конечной нормой

$$
\|B\|_{2}=\left(\sum_{i=1}^{\infty}\left\|B e_{i}\right\|^{2}\right)^{1 / 2}
$$

Ядерным называется оператор $B$, представимый суммой $B=\sum_{i=1}^{\infty} C_{i} D_{i}$ попарных произведений операторов Гильберта-Шмидта, имеющий конечную ядерную норму. Ядерной нормой $\|B\|_{1}$ называется инфимум по всем таким представлениям сумм $\sum_{i=1}^{\infty}\left\|C_{i}\right\|_{2} \cdot\left\|D_{i}\right\|_{2}$. Заметим, что если $C$ - линейный оператор, то $\|B C\|_{1} \leqslant\|B\|_{1} \cdot\|C\|$.

Суперследом әлемента $B$ пространства $N\left(E_{\Lambda}\right)$ всех ядерных операторов на $E_{\Lambda}$, наделенного нормой $\|\cdot\|_{1}$, называется величина $\operatorname{str} B=$ $\operatorname{tr}\left(B_{00}\right)-\operatorname{tr}\left(B_{11}\right)$ (см. [7, c. 93]).

Оператор $C$ назьвается суперсимметричным, если каковы бы ни были $X, Y \in E_{\Lambda}$ верно равенство $(C X, Y)=(X, C Y)$.

Пусть $D$ - линейный оператор, такой что $(D-\mathbf{1})$ - ядерный $(\mathbf{1}$ - тождественный оператор), и все элементы его матрицы $\check{D}$ принадлежат $\Lambda_{0}$. Детерминанmом $\operatorname{det} D$ оператора $D$ называется предел при $n \rightarrow \infty$ главных миноров порядка $n$ матрицы $\check{D}$ (ср. [22, гл. $4, \S 1]$, [23, гл. $11, \S 6])$. Если оператор $C$ таков, что $(C-\mathbf{1}) \in N\left(E_{\Lambda}\right)$, а $C_{11}$ обратим, то величина

$$
\operatorname{sdet} C=\operatorname{det}\left(C_{00}-C_{01} C_{11}^{-1} C_{10}\right) \operatorname{det} C_{11}^{-1}
$$

назьвается супердетерминантом оператора $C$ (ср. [7, с. 93]).

Теорема 1 (Лиувилля, cp. [7, с. 101]). Пусть $\varepsilon>0, A$-аналитическое отобрахсение (см. [3]) области $U=\left\{t \in \Lambda_{0}^{\mathbb{C}}|| t \mid<\varepsilon\right\}$ в пространство $N\left(E_{\Lambda}\right)$. Тогда в этой области существует решение задачи Коши $\left\{D^{\prime}(t)=A(t) D(t)\right.$, $\left.D(0)=D_{0}\right\}$, причем если $\left(D_{0}-\mathbf{1}\right) \in N\left(E_{\Lambda}\right)$, mо $(D(t)-\mathbf{1}) \in N\left(E_{\Lambda}\right)$ для всех $t \in U, u(\operatorname{sdet} D(t))^{\prime}=\operatorname{str} A(t) \cdot \operatorname{sdet} D(t)$. (Здесь берутся формальные производные степенных рядов.)

3. Функции и супермеры. Отображение $f$ открытого подмножества $U \subset F_{\Lambda}$ в суперпространство $G_{\Lambda}$ назьвается супердифферениируемым в точке $X$, если оно дифференцируемо по Фреше, причем его производная $f^{\prime}(X)$ принадлежит пространству $\operatorname{Hom}_{\Lambda}(F, G)$. Производная в точке $X$ от производной порядка $n-1$ (где 
$n \geqslant 2$ ) называется производной $f^{(n)}(X)$ порядка $n$ (ее можно считать элементом пространства $\left.\operatorname{Hom}_{\Lambda}\left(\widehat{\otimes}^{n} F, G\right)\right)$.

Если существует $n$-я производная $D^{n} f(X)$ по нечетным переменным, то она принадлежит пространству $\operatorname{Ahom}_{\Lambda}\left(\widehat{\otimes}^{n} F_{1}, G\right)$ антисимметричных операторов на $\left(\widehat{\otimes}^{n} F_{1}\right)_{\Lambda}$, которые можно считать $\Lambda$-линейньпи справа непрерывньпи операторами, действующими в пространстве $\left(\widehat{\wedge}^{n} F_{1}\right)_{\Lambda}$, пополненном в проективной топологии. (Здесь $\wedge^{n} F_{1}-n$-я внешняя степень пространства $F_{1}$, см. [24].)

Прямую сумму

$$
\sum_{n=0}^{\infty} \operatorname{Hom}_{\Lambda}\left(\widehat{\wedge}^{n} F_{1}, G\right)
$$

обозначим символом $\operatorname{Hom}_{\Lambda}\left(\widehat{\wedge} F_{1}, G\right)$, а подкласс ограниченных последовательностей в классе

$$
\prod_{n=1}^{\infty}\left(\widehat{\wedge}^{n} F_{1}\right)_{\Lambda}
$$

наделенный $\ell_{\infty}$-нормой, - символом $\left(\widehat{\wedge} F_{1}\right)_{\Lambda}$. Тогда на $\operatorname{Hom}_{\Lambda}\left(\widehat{\wedge} F_{1}, G\right) \times\left(\widehat{\wedge} F_{1}\right)_{\Lambda}$ определено естественное непрерьвное $G_{\Lambda}$-значное билинейное отображение

$$
T:(A, \nu) \mapsto \sum_{n=1}^{\infty} A_{n}\left(\nu_{n}\right),
$$

где $A=\left(A_{1}, \ldots, A_{n}, \ldots\right), \nu=\left(\nu_{1}, \ldots, \nu_{n}, \ldots\right)$.

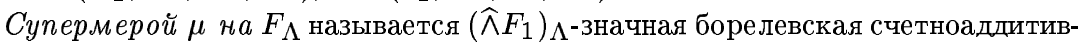
ная мера на $\left(F_{\Lambda}\right)_{0}$, имеющая ограниченную вариацию $v(\mu)$. Пусть $M_{b}\left(F_{\Lambda}\right)-$ множество всех супермер на $F_{\Lambda}$ с ограниченным носителем.

Для всякой функции $f: E_{\Lambda} \rightarrow G_{\Lambda}$, бесконечное число раз супердифференцируемой по нечетным переменным, и для любого $r>0$ определим на $\left(E_{\Lambda}\right)_{0}$ отображения

$$
\pi_{r}(f): x \mapsto\left(f(x), r D f(x), \ldots, r^{n} D^{n} \frac{f(x)}{n !}, \ldots\right), \quad \pi(f)=\pi_{1}(f) .
$$

Обозначим через $F_{r}\left(E_{\Lambda}, G_{\Lambda}\right)$ множество функций $f$, для которых $\pi_{r}(f)\left(\left(E_{\Lambda}\right)_{0}\right) \subset$ $\operatorname{Hom}_{\Lambda}\left(\widehat{\wedge} F_{1}, G\right)$. Пусть, кроме того, $F_{b, r}\left(E_{\Lambda}, G_{\Lambda}\right)$ - множество всех функций из класса $F_{r}\left(E_{\Lambda}, G_{\Lambda}\right)$, таких что отображение $\pi_{r}(f):\left(E_{\Lambda}\right)_{0} \rightarrow \operatorname{Hom}_{\Lambda}\left(\widehat{\wedge} F_{1}, G\right)$ непрерьвно и ограничено на ограниченных подмножествах, $F_{b}\left(E_{\Lambda}, G_{\Lambda}\right)=F_{b, 1}\left(E_{\Lambda}, G_{\Lambda}\right)$.

ЗАмЕчание 1 . Произведение двух функций из классов $F_{b, 16 r}\left(E_{\Lambda}, G_{\Lambda}\right)$ и $F_{b, 16 r}\left(E_{\Lambda}, \Lambda_{0}\right)$ соответственно является элементом класса $F_{b, r}\left(E_{\Lambda}, G_{\Lambda}\right)$.

ЗАмечание 2. Функции $f_{Y}: X \mapsto \exp \{i(X, Y)\}, g_{C}: X \mapsto(C X, X)$ и $h_{C}:$ $X \mapsto \exp \{(C X, X)\}$ принадлежат пространствам $F_{b, r}\left(E_{\Lambda}, \Lambda_{0}^{\mathbb{C}}\right)$, каковы бы ни были элементы $Y \in E_{\Lambda}, C \in \operatorname{Hom}_{\Lambda}\left(E, E^{\mathbb{C}}\right), r>0$. (Здесь $F^{\mathbb{C}}$ - комплексификация $F$.)

Tеорема 2. Eсли $f \in F_{b}\left(E_{\Lambda}, G_{\Lambda}^{\mathbb{C}}\right), \mu \in M_{b}\left(E_{\Lambda}\right)$, то функиия

$$
\pi(f):\left(E_{\Lambda}\right)_{0} \rightarrow \operatorname{Hom}_{\Lambda}\left(\widehat{\wedge} E_{1}, G^{\mathbb{C}}\right)
$$


интегрируема в смысле Бартла (см. [25]) по супермере $\mu$ относительно билинейного отображения $T$.

Величина билинейного интеграла

$$
\int_{\left(E_{\Lambda}\right)_{0}} T(\pi(f)(X), \mu(d X))
$$

назьвается интегралом $\int f(X) \mu(d X)$ функиии $f$ по супермере $\mu$.

Преобразованием Фурье супермеры $\mu \in M_{b}\left(E_{\Lambda}\right)$ называется функция

$$
\widetilde{\mu}: E_{\Lambda} \mapsto \Lambda^{\mathbb{C}}, X \mapsto \int \exp \{i(X, Y)\} \mu(d Y) .
$$

ТЕорема 3 (о дифференцировании под знаком интеграла). Пусть $U-o m$ крытое подмнохсество $E_{\Lambda}, u$ функиия $g: U \times E_{\Lambda} \rightarrow G_{\Lambda}^{\mathbb{C}}$ такова, что каково бы ни било $k=0,1, \ldots, n+1$, отображсение $g_{k}: X \mapsto g_{x}^{(k)}(X, \cdot)$, $U \rightarrow F_{b}\left(E_{\Lambda}, \operatorname{Hom}_{\Lambda}\left(\widehat{\otimes}^{n} E, G^{\mathbb{C}}\right)\right)\left(\right.$ здесь $g_{x}^{(k)}-k$-я производная по первому аргументу) локально ограничено. Тогда если $\mu \in M_{b}\left(E_{\Lambda}\right)$, то функиия $f: X \mapsto \int g(X, Y) \mu(d Y), U \rightarrow G_{\Lambda}^{\mathbb{C}}$, имеет производные до порядка $n$ включительно, причем $f^{(k)}(X)=\int g_{x}^{(k)}(X, Y) \mu(d Y)(n \geqslant 1, k=1,2, \ldots, n)$.

ДОКАЗАТЕЛЬСТВО Проводится по индукции.

4. Гармонический осциллятор. Для ядерного оператора $B$ в $E_{\Lambda}$ и дважды супердифференцируемой функции $f$ определен суперлапласиан $\Delta_{B} f=\operatorname{str}\left(f^{\prime \prime} B J\right)$, где $J:(x, \theta, \bar{\theta}) \mapsto(x, \theta,-\bar{\theta})$ - оператор в $E_{\Lambda}$.

TЕОРема 4. Пусть $B, C$ - суперсимметричные операторы в $E_{\Lambda}$, причем $B$ - ядерныц $; \mu_{0} \in M_{b}\left(E_{\Lambda}\right), \alpha \in \Lambda_{0}^{\mathbb{C}}, \delta=\pi\left(2(\|B\| \cdot\|C\|)^{1 / 2}|\alpha|\right)^{-1}$. Тогда в классе функиий $f:\left\{t \in \Lambda_{0}^{\mathbb{C}}|| t \mid \leqslant \delta\right\} \times E_{\Lambda} \rightarrow \Lambda^{\mathbb{C}}$ разрешима задача Коши для уравнения

$$
f_{t}^{\prime}(t, X)=\frac{\alpha}{2}\left(-\Delta_{B} f(t, X)+(C X, X) f(t, X)\right)
$$

с начальным условием $f(0, \cdot)=\widetilde{\mu}_{0}(\cdot)$. Причем существует решение вида $f(t, X)=\int G(t, X, Y) \mu_{0}(d Y)$, əдe

$$
G(t, X, Y)=s(t, Y) \exp \left\{\frac{(Q(t) X, X)}{2}+i(R(t, Y), X)\right\},
$$

а функции s, $Q$ и $R$ определяются равенствами:

$$
\begin{gathered}
s=\operatorname{sdet}^{-1 / 2}(\operatorname{ch}(\alpha t \sqrt{C B})) \exp \left\{\frac{1}{2} B\left(\frac{\operatorname{th}(\alpha t \sqrt{C B})}{\sqrt{C B}} Y, Y\right)\right\}, \\
Q(t)=\frac{\operatorname{th}(\alpha t \sqrt{C B})}{\sqrt{C B}} C, \quad R(t, Y)=\operatorname{ch}^{-1}(\alpha t \sqrt{C B}) Y .
\end{gathered}
$$

(Четные функиии от $\sqrt{C B}$ определяются с помощью ряда Маклорена.)

ДокАЗАТЕльство. Выполнение начальных условий очевидно. Непосредственной подстановкой убеждаемся, что функция $G(\cdot, \cdot, Y)$ вида $(2)$ будет решением уравнения (1) с начальными условиями $G(0, \cdot, Y)=\exp \{i(\cdot, Y)\}$ тогда и только 
тогда, когда функции $s, Q$ и $R$ аргумента $t$ ( $Y$ - параметр) будут решениями системы уравнений

$$
s_{t}^{\prime}=s \cdot \frac{\alpha(\operatorname{str}(Q B)+(B R, R))}{2}, \quad R_{t}^{\prime}=-\alpha Q B R, \quad Q_{t}^{\prime}=\alpha(C-Q B Q)
$$

с начальными условиями $\{s(0, Y)=1, Q(0)=0, R(0, Y)=Y\}$. Непосредственная проверка с использованием теоремы 1 показывает, что формулы, приведенные в формулировке теоремы, действительно определяют решение этой системы. Доказательство завершается применением теоремы 3 .

ЗАмечАниЕ. Уравнению Шрёдингера соответствует значение $\alpha$, равное $-i$.

В заключение автор выражает благодарность О. Г. Смолянову за постановку задачи и внимание к работе.

Нижегородский государственньй университет

Поступило им. Н. И. Лобачевского 20.11 .95

\section{СПИСОК ЦИТИРОВАННОЙ ЛИТЕРАТУРЫ}

1. Глимм Дж., Джаффе А. Математические методы квантовой физики. М.: Мир, 1984. 2. Rogers A.// J. Math. Phys. 1981. V. 22. № 5. Р. 939-945. 3. Владимиров В. С., Волович И. В. // ТМФ. 1984. Т. 59. №1. С. 3-27. 4. Смолянов О. Г., Шавгулидзе Е. Т. // УМН. 1986. Т. 41. № 4. С. 164-165. 5. Владимиров В. С., Волович И. В. // ТМФ. 1984. Т. 60. №2. С. 169-198. 6. Хренников А. Ю. // УМН. 1988. Т. 43. № 2. С. 87-114. 7. Березин Ф. А. Введение в алгебру и анализ с антикоммутирующими переменными. М.: Изд-во МГУ, 1983. 8. Хренников А. Ю. // ТМФ. 1987. Т. 72. №3. С. 24-34. 9. Смолянов О. Г., Шавгулидзе Е. Т. // Докл. АН СССР. 1988. Т. 299. № 4. С. 816-821. 10. Хренников А. Ю. // Дифференц. уравнения. 1988. Т. 24. № 12. С. 2144-2153. 11. Смолянов О. Г., Шавгулидзе Е. Т. // Докл. АН СССР. 1990. Т. 309. С. 545-550. 12. Хренников А. Ю. // Матем. заметки. 1990. Т. 48. № 2. С. 114-122. 13. Маслова Е. В. // Вестн. МГУ. Сер. 1. Матем., мех. 1990. Т. 6. С. 72-75. 14. Rogers A. // J. Geom. Phys. 1993. V. 2. №1-4. P. 491-505. 15. Хренников А. Ю. // Изв. АН СССР. Сер. матем. 1990. Т. 54. № 3. С. 576-606. 16. Хренников А. Ю. // Дифференц. уравнения. 1992. Т. 28. № 8. С. 1434-1443. 17. Хренников А. Ю. // УМН. 1984. Т. 39. №1. С. 163-164. 18. Галкин О. Е. // Сб. "Исследования по теории функций". Н. Новгород: ННГУ, 1992. С. 1113. 19. Робертсон А. П., Робертсон В. Дж. Топологические векторные пространства. М.: Мир, 1967. 20. Шефер Х. Топологические векторные пространства. М.: Мир, 1971. 21. Хренников А. Ю. // Докл. АН СССР. 1991. Т. 321. № 2. С. 298-301. 22. Гохберг И. Ц., Крейн М. Г. Введение в теорию линейных несамосопряженных операторов в гильбертовом пространстве. М.: Наука, 1965. 23. Данфорд Н., Шварц Дж. Т. Линейные операторы. Т. 2. Спектральная теория. Самосопряженные операторы в гильбертовом пространстве. М.: Мир, 1966. 24. Бурбаки Н. Алгебpa. Алгебраические структуры. Линейная и полилинейная алгебра. М.: Физматгиз, 1962. 25. Bartle R. G. // Studia Math. 1956. V. 15. № 3. P. 337-352. 